Memorias del VII Encuentro Nacional de Experiencias en la Enseñanza de la Biología y la Educación Ambiental y 11 Congreso Nacional de Investigación en la Enseñanza de la Biología

\title{
EL DIARIO DE CAMPO \\ DEL DES-CONOCIMIENTO AL RE-CONOCIMIENTO DE LA DIVERSIDAD BIOLÓGICA DE SAN ZENÓN (MAGDALENA)
}

\section{Tania Ximena Salcedo Gómez ${ }^{1}$}

\section{Resumen}

Este trabajo centra su atención en el diario de campo, como una estrategia de enseñanza y aprendizaje individual y colectivo, sobre la vida y lo vivo. Para ello se realiza un estudio con los estudiantes de décimo y once, de la Institución Educativa Departamental Gerardo Valencia Cano; en el que, a través de la observación, la indagación y el registro en el diario de campo (de los conocimientos acerca de la fauna, la flora, y algunos aspectos de la historia y la población), se pudo dar un paso entre el des-conocimiento, al re-conocimiento de la diversidad y del sí mismo; promoviendo al mismo tiempo el desarrollo de algunas habilidades y contribuyendo en la superación de algunas falencias.

Palabras Clave: diario de campo, diversidad, enseñanza de la biología, estrategia de aprendizaje

\section{Abstract}

This work focuses on the field diary as a teaching and learning strategy individually and collectively, about life and the living. This is a study with ten and eleven students, Department of School Gerardo Valencia Cano, in which, through observation, inquiry and journaling field (of knowledge about the fauna, flora, and some aspects of the history and people), it was possible to step between the lack of knowledge, to the recognition of diversity and himself while promoting the development of some skills and contributing in overcoming some shortcomings.

Key Words: Field diary, diversity, biology teaching, learning strategy

\section{Introducción}

Los sujetos están constituidos por saberes, significados y sentidos, que pueden ser develados a través del uso de herramientas y estrategias pedagógicas, tales como el diario de campo (Albertín, 2007). Ésta es una herramienta, que permite al estudiante auscultar entre sus propias vivencias y ambientes, al mismo tiempo que

${ }^{1}$ Estudiante de Licenciatura en Biología. Universidad Pedagógica Nacional tiacosa-29@hotmail.com 
Bio-grafia Escritos sobre la Biologia y su Enseñanza.

Edición Extra-Ordinaria. ISSN 2027-1034 P.p 849-857

Memorias del VII Encuentro Nacional de Experiencias en la Enseñanza de la Biología y la Educación Ambiental y 11 Congreso Nacional de Investigación en la Enseñanza de la Biología

desarrolla actitudes de investigación y habilidades tales como: la descripción, el análisis y la argumentación (Porlán, 2008; Taylor y Bogdan, 1987)

En la enseñanza de la biología y especialmente de la biodiversidad y la conservación, el uso del diario de campo, se convierte en una posibilidad para que el estudiante a través de sus observaciones, descripciones, reflexiones y cuestionamientos, pueda llegar paulatinamente a un proceso de re-conocimiento, en donde se re-evalúen los pensamientos y acciones sobre su entorno; lo cual es muy importante para que el individuo se vuelva más autónomo en cuanto a sus procesos de aprendizaje, como también hacia la construcción de sus perspectivas en relación a la vida y a lo vivo (Albertín, 2007; Guber, 2001; Roa y Vargas, 2009)

Conocer y conservar lo vivo, requiere reconocer todo un contexto socio-cultural, en el que emergen prácticas, conocimientos, vivencias, emociones y valores al interior de la comunidad; debemos entender que la diversidad biológica se encuentra íntimamente relacionada con la gente y su cultura, especialmente en contextos rurales, como en el municipio de San Zenón - Magdalena (Primack, 2001). En dicho sentido, el diario de campo, emerge como una posibilidad real para que tanto el maestro de biología, como el estudiante, puedan llegar a comprender paulatinamente los elementos que configuran su ambiente, así como las relaciones que acaecen en el.

Adicionalmente, la interlocución que se da con los otros y consigo mismo por medio de los registros del diario de campo, conllevan hacia la construcción de escenarios de debate y análisis, en los que el sujeto puede llegar a cuestionarse, confrontarse, y reconstruir el conocimiento previo (Porlán, 2008; Albertín, 2007). En tanto, el maestro debe orientar al estudiante para que construya un cuaderno de campo, que dé cuenta de algo, datos que en conjunto permitan entender y entenderse, ya sea de manera individual o colectiva (Porlán, 2008; Albertín, 2007).

Debemos tener en cuenta como maestros que los procesos de aprendizaje están mediados por múltiples elementos. Como veremos a lo largo de éste artículo, no solo basta con presentar el diario de campo, como un elemento autosuficiente, puesto su inmersión en el aula, es todo un proceso de asimilación por parte del estudiante; requiere de generar hábitos de escritura, escucha, y participación; de construir preguntas, de generar hipótesis, de analizar, de replantearse constantemente, de movilizar el pensamiento (Porlán, 2008; Roa y Vargas, 2009).

En conclusión el diario de campo es una propuesta de de-construcción individual y colectiva sobre los pensamientos y las prácticas que emergen dentro y fuera del aula, así como de los procesos de enseñanza y aprendizaje, tanto de la biología, como de la vida misma. 
Memorias del VII Encuentro Nacional de Experiencias en la Enseñanza de la Biología y la Educación Ambiental y 11 Congreso Nacional de Investigación en la Enseñanza de La Biología

\section{Metodología}

En el primer semestre del año 2012, entre los meses de febrero a Junio, los estudiantes de grado decimo y once, de la Institución Educativa Departamental Gerardo Valencia Cano, ubicada en el municipio de San Zenón (Magdalena); empezaron a llevar un diario de campo, como parte de un trabajo investigativo en el área de biología. En ese diario, debían registrar las descripciones del clima, la fauna, la flora, y otros elementos que emergían durante las salidas de campo y la investigación individual.

Para empezar con el trabajo de diario de campo, los estudiantes y el docente, abordaron una lectura en la que se mencionaban los elementos que configuraban un diario de campo; con el fin de enseñar algunos de los parámetros que se deben tener en cuenta, a la hora de hacer uso de esta herramienta. Posteriormente se mostraron algunos ejemplos tanto de la etnografía en Colombia, como de los diarios de campo de algunos naturalistas.

Los estudiantes realizaron dos entregas de cuaderno de campo; en las que se consignaron las dos salidas de campo que se realizaron a la ciénaga, la cienaguita, y a los alrededores de la cabecera municipal. Así mismo se presentaron los datos de las investigaciones individuales de la flora y la fauna del municipio de San Zenón (Magdalena), y algunos aspectos históricos y descriptivos de la población. Es preciso mencionar que en la primera entrega del diario de campo se hicieron algunas orientaciones en relación al manejo adecuado de esta herramienta, así como a algunas estrategias para mejorar las observaciones; de tal forma que en la segunda entrega se hiciera una revisión colectiva del diario de campo y se pudieran debatir y analizar algunos aspectos registrados.

El análisis de los diarios de campo, se realizo de forma cualitativa, a través de 3 categorías: la descripción, la redacción y el dibujo. Para ello se tomaron como ejemplo algunos diarios de campo, en los que se evidencian algunas de las tendencias principales en la primera y segunda entrega del diario de campo. Así mismo se evaluaron algunas de las observaciones hechas por los estudiantes, durante la socialización.

\section{Resultados Y Discusión}

\section{Primera entrega}


Memorias del VII Encuentro Nacional de Experiencias en la Enseñanza de la Biología y la Educación Ambiental y 11 Congreso Nacional de Investigación en la Enseñanza de la Biología
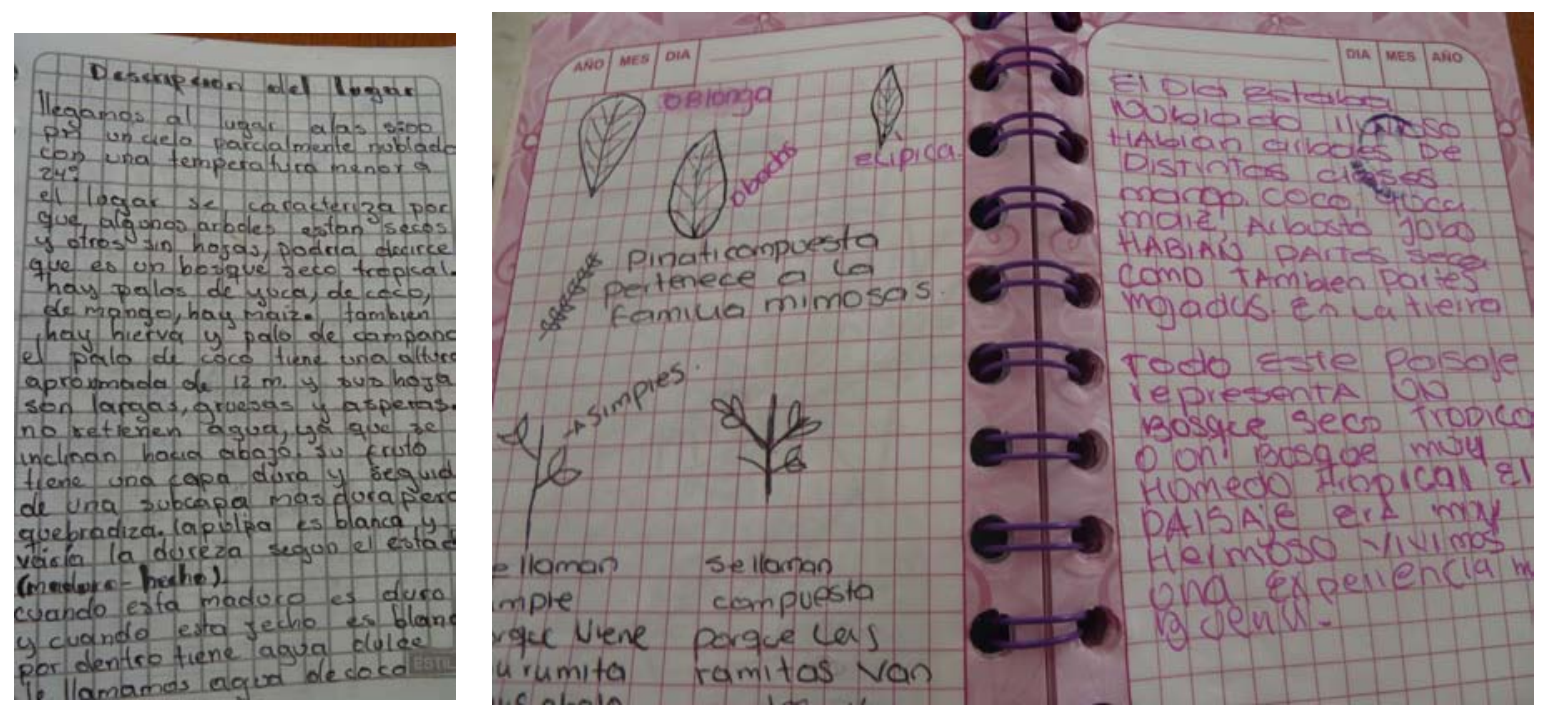

IMAGEN 1 (centro), 2 (Izquierda superior)

A través de los 34 estudiantes que presentaron su diario de campo (de los 54 que conformaban el grado décimo y once), se lograron evidenciar varios elementos, en cuanto a las descripciones, redacción e ilustraciones. En el primer caso, las observaciones y apuntes, fueron muy someros, se limitaron a escribir lo que les referenciaba el profesor, haciendo uso de términos biológicos, que no eran claros, como por ejemplo "Bosque seco tropical", "hojas simples y compuestas" y/o "especie" (VER IMAGEN 1).

Las descripciones propias fueron muy limitadas, aunque los pocos intentos que se registraron, no solo hacían un buen uso del lenguaje, sino que además trataban de dar a conocer el significado que tenían para ellos ciertas expresiones, como "hecho" y "maduro" (VER IMAGEN 2); lo que fue y es totalmente relevante a la hora de generar puentes de comunicación bidireccional y procesos de aprendizaje en la biología.

La redacción por su parte, presentaba serías falencias de coherencia, puntuación y ortografía en el $90 \%$ de los casos; la gran mayoría de las ideas se encontraban sueltas y no lograban generar relaciones entre los apuntes consignados (VER IMAGEN 3).

Existían dificultades para entrelazar el conocimiento y las observaciones, y por ende para generar preguntas y descripciones más detalladas y comprensibles. Desafortunadamente, estas y otras falencias, se han convertido en el común denominador de los estudiantes, siendo un aspecto cuestionable sobre el qué y el cómo se ésta llevando a la educación; sin mencionar cuál es el compromiso de la 
Memorias del VII Encuentro Nacional de Experiencias en la Enseñanza de la Biología y la Educación Ambiental y II Congreso Nacional de Investigación en la Enseñanza de la Biología

sociedad (padres, maestros, y otros) en los procesos de formación. Pareciera además, que estas falencias solo le competen al docente de español, pero en realidad están truncando cualquier proceso que se quiera realizar desde las diferentes asignaturas, de tal forma que es preciso, realizar un trabajo conjunto, en el que el estudiante pueda superar paulatinamente estas dificultades; siendo el diario de campo una gran estrategia para lograrlo.

Por otro lado, las ilustraciones que realizaron los estudiantes en sus diarios de campo, dejaban entrever varios elementos. Los dibujos funcionaron como fuente de referencia y descripción; al mismo tiempo que permitían al lector identificar dos tipos de habilidades, la observación y el dibujo. Cada uno de ellos, fundamental para generar mejores procesos de aprendizaje de la biología y de la vida misma. Al mismo tiempo, se pudo observar que el estudiante a través del dibujo, lograba plasmar y reflejar los elementos con los que tenía mayor relación o los que presentaban algún significado para él; consolidando esta fuente, como un medio, para conocer y reconocer, al sujeto y su ambiente (VER ANEXOS 1, 2 Y 3)

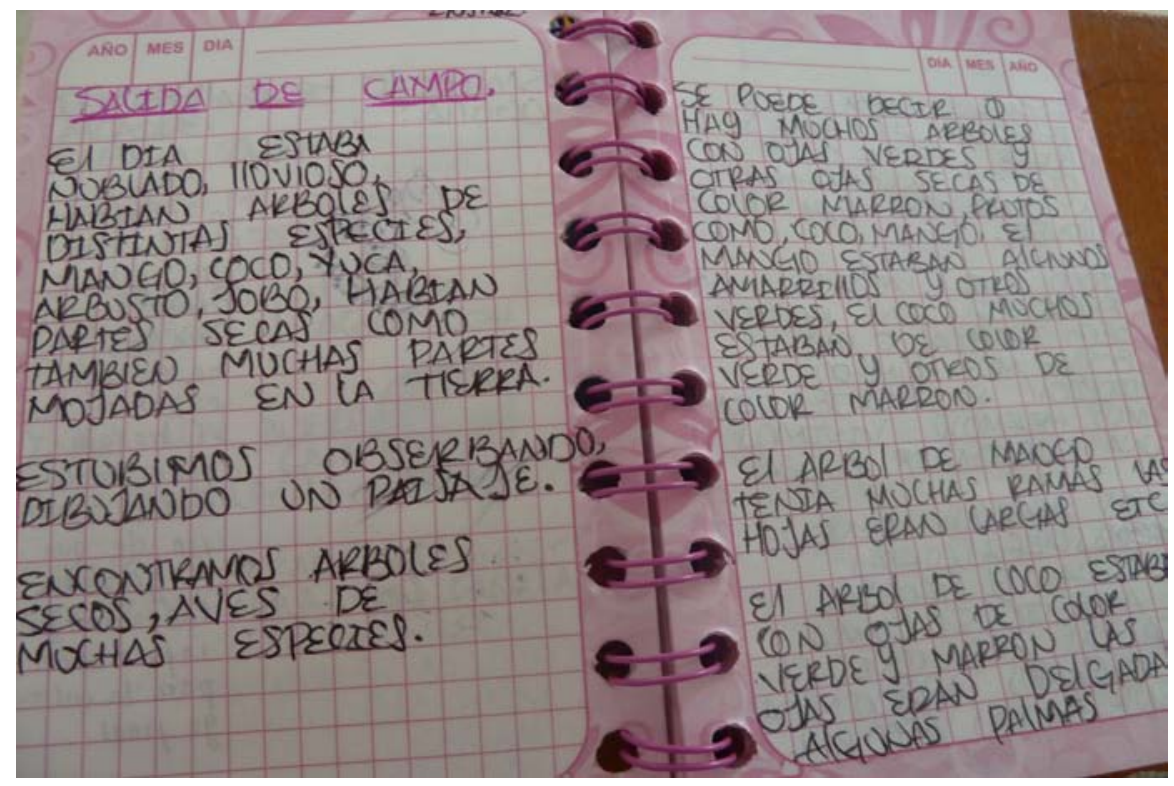

(IMAGEN 3)

Cabe señalar, que la ilustración consignada en el diario de campo, no solo es una fuente que permite develar habilidades, significados y percepciones; sino que además, dependiendo del grado de precisión y detalle que tenga la ilustración, se convierte en una fuente para inferir distintos aspectos como la riqueza de especies, las relaciones ecológicas y los periodos climáticos, así como otros aspectos muy importantes para describir y entender lo vivo. 
Memorias del VII Encuentro Nacional de Experiencias en la Enseñanza de la Biología y la Educación Ambiental y 11 Congreso Nacional de Investigación en la Enseñanza de la Biología

\section{Segunda entrega}

En esta entrega, los 16 estudiantes que presentaron sus cuadernos de campo, mostraron avances muy importantes en el conocimiento de su municipio, gran parte de ellos realizaron descripciones mucho más precisas y detalladas de algunas de las plantas de San Zenón. Otros, simplemente se limitaron a copiar descripciones de internet, y otros descubrieron aspectos muy interesantes sobre la historia y su comunidad (Ver las imágenes $4,5,6$ y 7 )

Por otro lado, durante la revisión colectiva de los diarios de campo, se lograron evidenciar y discutir algunos de los cambios que ha tenido la comunidad a lo largo del tiempo, principalmente en relación a los recursos y al conocimiento de la biodiversidad; los estudiantes ponian de manifiesto que habían muchos aspectos que no conocian de sus entornos, especialmente en relación a los nombres comunes de las plantas, a sus usos y a las relaciones con la gente y sus actividades económicas. Algunos mostraban con sus expresiones, emociones de desconcierto y de curiosidad, puesto se dieron cuenta de que a pesar de vivir en el municipio, habían muchas cosas que no conocian de él, y que hacian parte de su identidad cultural.
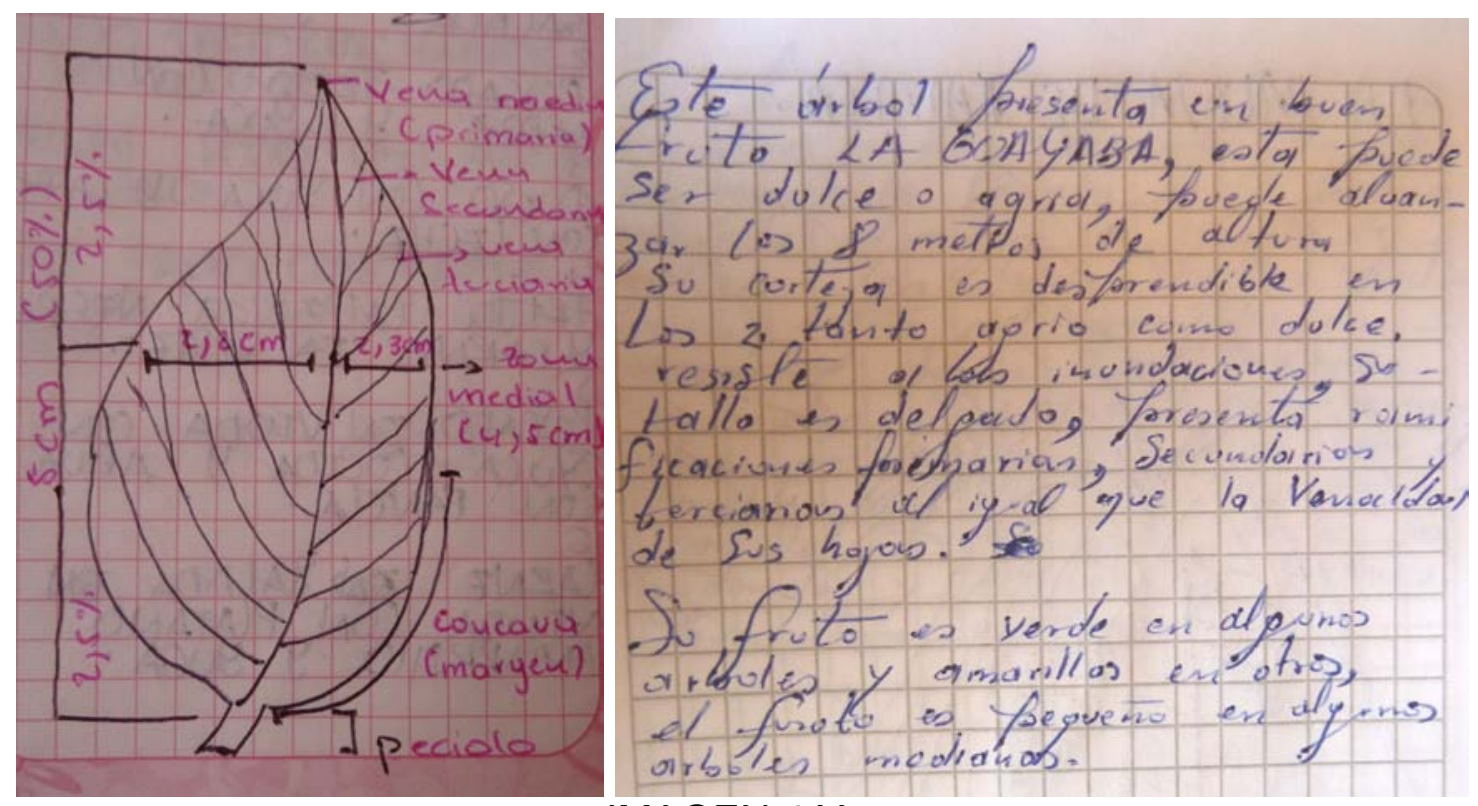

IMAGEN 4 Y 5 
Memorias del VII Encuentro Nacional de Experiencias en la Enseñanza de la Biología y la Educación Ambiental y 11 Congreso Nacional de Investigación en la Enseñanza de la Biología

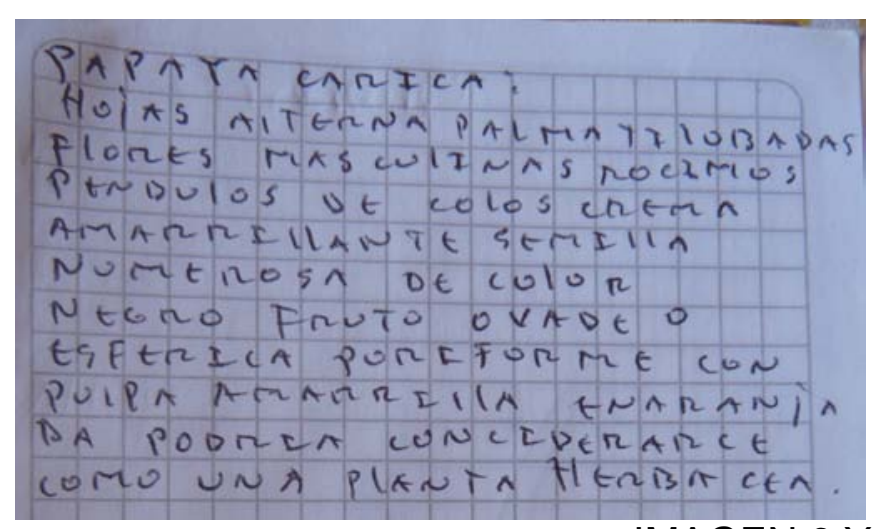

IMAGEN 6 Y 7

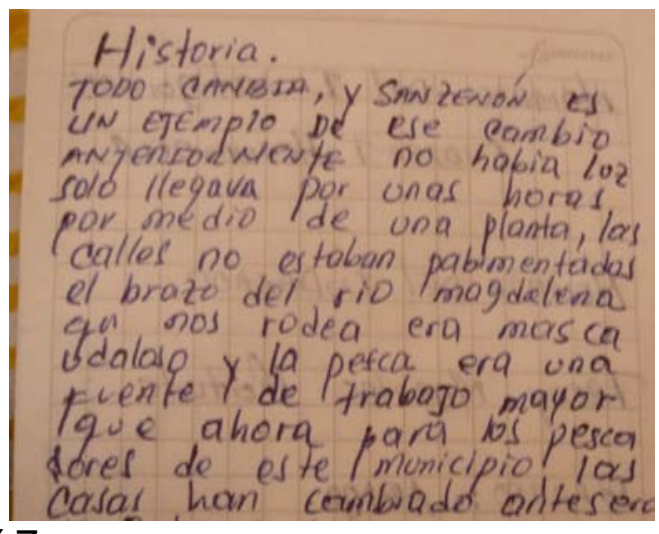

\section{CONCLUSIONES}

A través del diario de campo, el maestro y el estudiante pueden plasmar los conocimientos que tienen acerca de la biodiversidad y el ambiente en el que se desenvuelven; de tal forma que a través del análisis individual y grupal de los datos que allí se consignan, el sujeto puede llegar a cuestionarse, confrontarse, y reconstruir el conocimiento previo.

El diario de campo reformula las formas de enseñar y aprender biología, especialmente en los contextos rurales, en donde la mayoría de prácticas culturales se encuentran directamente relacionadas con los entornos naturales. De tal forma que no se trata de satanizar las prácticas o los discursos populares a través de la ciencia, sino más bien de generar un dialogo de saberes, que a través de la disertación permitan al sujeto comprender y aprender desde los significados, las experiencias y las reflexiones del ambiente en el que se desenvuelven.

El diario de campo pone en cuestión lo que el sujeto cree que sabe, lo confronta con su propia cultura, lo invita a redimensionar el mundo en el que habita, desde los valores, las prácticas, las costumbres, las palabras... lo que es como sujeto socialmente indivisible. Es quizás esta confrontación la que permite, que el sujeto y la comunidad se hagan más consientes de lo que tienen desde los valores que le han atribuido a los elementos que giran en torno a ellos, puesto hacen parte de sus cotidianidades, de su vida misma.

La transformación que se logra a través de la investigación, las salidas de campo y la consignación de los datos en un diario de campo; radica en la revalorización que empiezan a generar los sujetos por su ambiente. Indiscutiblemente estos procesos requieren un seguimiento constante de interacción investigativa y 
Memorias del VII Encuentro Nacional de Experiencias en la Enseñanza de la Biología y la Educación Ambiental y 11 Congreso Nacional de Investigación en la Enseñanza de la Biología

participativa con la comunidad, pero lo más importante es que cada diario de campo constituye una real experiencia de vida, de percepción y es propio, por lo que se hace muy importante para el sujeto que lo construye.

En síntesis el diario de campo y todos los elementos que giran en torno a su construcción, no solo potencian habilidades, muestran falencias, recuperan conocimientos y re-dimensionan culturas, sino que además problematizan al sujeto y ese es el mayor logro que puede adquirirse en un proceso de enseñanza, puesto el aprendizaje que se genera comienza a ser más significativo para el sujeto, en tanto radica de sus propios cuestionamientos y reflexiones.

Particularmente este estudio, nos demuestra cómo una simple herramienta como lo es el diario de campo puede convertirse en una estrategia para re-conocer, deconstruyendo imaginarios, sentidos, valores, saberes e incluso emociones con respecto al ambiente; convirtiéndose en un opción valiosa para el aprendizaje de la biología, y a futuro para la construcción de una sociedad más crítica y participativa.

\section{Bibliografía}

Albertín, P., (2007). La formación reflexiva como competencia profesional. Condiciones psicosociales para una práctica reflexiva. El diario de campo como una herramienta. Revista de enseñanza universitaria. 30, 7-18.

Guber, R. (2001). La etnografía: método, campo y reflexividad. Enciclopedia Latinoamericana de sociocultura y comunicación. Grupo editorial Norma.

Porlán, R. (2008). El diario de clase y el análisis de la práctica. Sevilla. Junta de Andalucía, Consejería de Educación y Ciencia.

Primack, R. (2001). Fundamentos de conservación biológica: perspectivas latinoamericanas. Fondo de cultura económica

Roa, P., Vargas, C. (2009). El cuaderno de campo como estrategia de enseñanza en el departamento de biología de la UPN. Bio-grafía: escritos sobre la biología y su enseñanza, 3, 80 - 91.

Taylor, S., Bogdan, R. (1987). Introducción a los métodos cualitativos de investigación. Barcelona: Paidós.

\section{Anexos}


Bio-grafia Escritos sobre la Biología y su Enseñanza.

Edición Extra-Ordinaria. ISSN 2027-1034 P. p 849-857

Memorias del VII Encuentro Nacional de Experiencias en la Enseñanza de la Biología y la Educación Ambiental y II Congreso Nacional de Investigación en la Enseñanza de la Biología
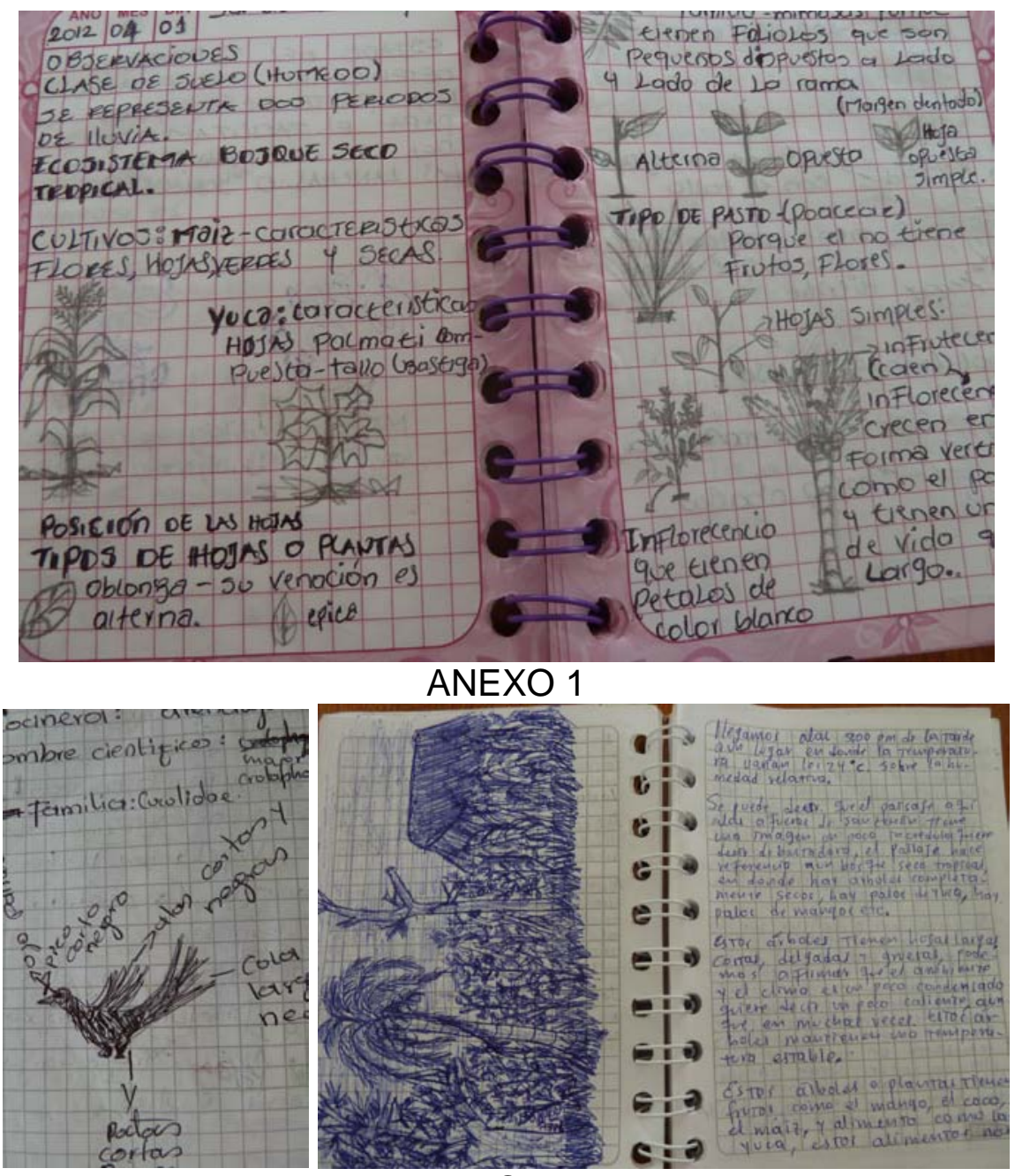

ANEXO 2 Y 3 